QUARTERLY OF APPLIED MATHEMATICS

VOLUME LXVI, NUMBER 4

DECEMBER 2008, PAGES 771-779

S 0033-569X(08)01085-4

Article electronically published on September 26, 2008

\title{
TRANSPORT OF HEAT AND MASS IN A FLUID WITH VANISHING MOBILITY
}

\author{
BY \\ CATHERINE CHOQUET
}

Université P. Cézanne, FST, LATP-CNRS UMR 6632, Case Cour A, 13397 Marseille Cedex 20,

France

\begin{abstract}
We study a model describing the compressible displacement of a mixture in a porous medium. The transport of heat and mass is described by a nonlinear, fully coupled and degenerate parabolic system. Using a series of compensated compactness and convexity arguments, we prove the existence of relevant weak solutions.
\end{abstract}

1. Introduction and main result. We consider the displacement of a mixture transported by a compressible flow in a porous medium. Let $\Omega$ be a domain of $\mathbb{R}^{N}$, $N \leq 3$, with $\mathcal{C}^{1}$ boundary $\Gamma$. The unit exterior normal is denoted by $\nu$. The time interval of interest is $(0, T), T>0$. We set $\Omega_{T}=\Omega \times(0, T)$ and $\Gamma_{T}=\Gamma \times(0, T)$. We denote by $p$ the pressure, by $\theta$ the temperature and by $\underline{u}$ the Darcy velocity of the fluid. Due to the mass and energy conservation principles ( $c f .63,6]$, , the flow is governed in $\Omega_{T}$ by

$$
\begin{aligned}
& \phi(x) \partial_{t} p+\operatorname{div}(\underline{u})=q^{i}-q^{s}, \quad \underline{u}=-\kappa(x, \theta) \nabla p, \\
& \phi(x) \partial_{t} \theta+\underline{u} \cdot \nabla \theta-\operatorname{div}(\mathcal{D}(\underline{u}) \nabla \theta)+q^{i} \theta=q^{i} .
\end{aligned}
$$

The second part of Eq. (1.1) is the classical Darcy law for porous media. The porosity of the medium is given by the function $\phi$. We assume that it belongs to $W^{1, \infty}(\Omega)$ and that there exist some reals $\phi_{-}$and $\phi_{+}$such that

$$
0<\phi_{-} \leq \phi(x) \leq \phi_{+} \text {a.e. in } \Omega \text {. }
$$

The function $\kappa$ describes the mobility of the fluid. It is defined by

$$
\kappa(x, \theta)=\frac{k(x)}{\mu(\theta)}
$$

where $k(x)$ is the permeability of the medium and $\mu$ is the viscosity of the fluid. The two main difficulties of the problem are connected with the mobility. On the one hand it

Received July 16, 2007.

2000 Mathematics Subject Classification. 35K60, 35K65, 35B40, 76S05, 35K57.

Key words and phrases. Nonlinear degenerate parabolic system; compensated compactness; miscible compressible displacement; porous media.

E-mail address: c.choquet@univ-cezanne.fr 
induces a strong coupling in the system because the viscosity is temperature dependent. On the other hand we admit here the possibility of a vanishing mobility (see Remark 1.3 below for physical motivations). If the admissible range of temperature for the model is $(0,1)$, we thus only assume that

$$
\begin{aligned}
& \kappa \in L^{\infty}(\Omega \times(0,1)) \text { with } \kappa(x, \theta) \geq 0 \text { a.e. } x \in \Omega, \\
& \nabla_{x}\left(\kappa(x, \theta)^{1 / 2}\right)=\left(\kappa^{-1 / 2} \nabla_{x} \kappa\right)(x, \theta) / 2 \in\left(L^{2}(\Omega)\right)^{N}, \quad \forall \theta \in(0,1) . \\
& D_{\theta}\left(\kappa(x, \theta)^{1 / 2}\right)=\left(\kappa^{-1 / 2} D_{\theta} \kappa\right)(x, \theta) / 2 \in\left(L^{2}(\Omega)\right)^{N},
\end{aligned}
$$

Considering a symmetric positive tensor of permeability $k$ brings no additional difficulty. Because of the vanishing mobility, the pressure equation (1.1) is of degenerate parabolic type. It is strongly coupled with (1.2). The diffusion effects are indeed modeled by the tensor

$$
\mathcal{D}(\underline{u})=\phi\left(D_{m} I d+D_{p}(\underline{u})\right)=\phi\left(D_{m} I d+|\underline{u}|\left(\alpha_{l} \mathcal{E}(\underline{u})+\alpha_{t}(I d-\mathcal{E}(\underline{u}))\right)\right),
$$

where $\mathcal{E}(\underline{u})_{i j}=\underline{u}_{i} \underline{u}_{j} /|\underline{u}|^{2}, \alpha_{l}$ and $\alpha_{t}$ are the longitudinal and transverse dispersion constants and $D_{m}$ is the molecular diffusion. The diffusion tensor satisfies the following properties:

$$
\mathcal{D}(\underline{u}) \xi \cdot \xi \geq \phi_{-}\left(D_{m}+\alpha_{t}|\underline{u}|\right)|\xi|^{2}, \quad|\mathcal{D}(\underline{u}) \xi| \leq \phi_{+}\left(D_{m}+\alpha_{l}|\underline{u}|\right)|\xi|, \quad \forall \xi \in \mathbb{R}^{N} .
$$

System (1.1)-(1.2) is completed by the following initial and boundary conditions:

$$
\begin{aligned}
& \underline{u} \cdot \nu=0 \quad \text { on } \Gamma_{T}, \quad p(x, 0)=p_{o}(x) \quad \text { in } \Omega, \\
& \mathcal{D}(\underline{u}) \nabla \theta \cdot \nu=0 \quad \text { on } \Gamma_{T}, \quad \theta(x, 0)=\theta_{o}(x) \quad \text { in } \Omega .
\end{aligned}
$$

The injection and production source terms $q^{i}$ and $q^{s}$ are nonnegative functions of $L^{2}(\Omega)$. The initial pressure $p_{o}$ belongs to $L^{2}(\Omega)$. We assume that the admissible range of temperature for the model is $(0,1)$. The initial temperature $\theta_{o} \in L^{\infty}(\Omega)$ thus satisfies $0 \leq \theta_{o}(x) \leq 1$ a.e. in $\Omega$.

We emphasize that the assumptions listed before are exactly those used by the authors who consider the nondegenerate problem (see Amirat et al. [1] who state the existence of weak solutions for Problem (1.1)-(1.2), (1.6)-(1.7) when the mobility is bounded below by a strictly positive constant). This paper is also a consequent improvement of 4 where we had to impose a nonnatural $L^{\infty}$ control on the pressure $p$. Our main result is the following.

Theorem 1.1. Assuming (1.3)-(1.5), Problem (1.1)-(1.2), (1.6)-(1.7) admits a weak solution $(p, \theta)$. The function $p \in L^{\infty}\left(0, T ; L^{2}(\Omega)\right)$ is a solution of (1.1), (1.6), verified in the space $L^{2}\left(0, T ;\left(H^{1}(\Omega)\right)^{\prime}\right)$; the Darcy velocity $\underline{u}$ belongs to $\left(L^{2}\left(\Omega_{T}\right)\right)^{N}$. The function $\theta \in L^{\infty}\left(\Omega_{T}\right) \cap L^{2}\left(0, T ; H^{1}(\Omega)\right)$ is such that $0 \leq \theta(x, t) \leq 1$ almost everywhere in $\Omega_{T}$.

The paper is organized as follows. We introduce a perturbation of Problem (1.1)-(1.2), (1.6)-(1.7) where a parameter $\varepsilon>0$ ensures a nondegenerate Darcy law. In Section 2, we derive some energy estimates of the corresponding solution $\left(p_{\varepsilon}, \theta_{\varepsilon}\right)$. Finally in Section 3 , we study the $\varepsilon$-limit of the perturbated problem to justify Theorem 1.1. To overcome the lack of estimate on the pressure gradient $\nabla p_{\varepsilon}$, we use compensated compactness arguments [8, 11]. 
Let $\varepsilon>0$. We define the following perturbated model in $\Omega_{T}$ :

$$
\begin{aligned}
& \phi(x) \partial_{t} p_{\varepsilon}+\operatorname{div}\left(\underline{u}_{\varepsilon}\right)=q^{i}-q^{s}, \underline{u}_{\varepsilon}=-\kappa_{\varepsilon}\left(x, \theta_{\varepsilon}\right) \nabla p_{\varepsilon}, \\
& \kappa_{\varepsilon}\left(x, \theta_{\varepsilon}\right)=\kappa\left(x, \theta_{\varepsilon}\right)+\varepsilon, \\
& \phi(x) \partial_{t} \theta_{\varepsilon}+\underline{u}_{\varepsilon} \cdot \nabla \theta_{\varepsilon}-\operatorname{div}\left(\mathcal{D}\left(\underline{u}_{\varepsilon}\right) \nabla \theta_{\varepsilon}\right)+q^{i} \theta_{\varepsilon}=q^{i}, \\
& \underline{u}_{\varepsilon} \cdot \nu=0 \quad \text { on } \Gamma_{T}, \quad p_{\varepsilon}(x, 0)=p_{o}(x) \quad \text { in } \Omega, \\
& \mathcal{D}\left(\underline{u}_{\varepsilon}\right) \nabla \theta_{\varepsilon} \cdot \nu=0 \quad \text { on } \Gamma_{T}, \quad \theta_{\varepsilon}(x, 0)=\theta_{o}(x) \quad \text { in } \Omega .
\end{aligned}
$$

System (1.8)-(1.9) is of parabolic type. For any fixed $\varepsilon>0$, Theorem 2 in [4 gives the following existence result.

Theorem 1.2. Problem (1.8)-(1.11) admits a weak solution $\left(p_{\varepsilon}, \theta_{\varepsilon}\right)$. The function $p_{\varepsilon} \in L^{\infty}\left(0, T ; L^{2}(\Omega)\right) \cap L^{2}\left(0, T ; H^{1}(\Omega)\right)$ is a solution of (1.8), (1.10) verified in the space $L^{2}\left(0, T ;\left(H^{1}(\Omega)\right)^{\prime}\right)$. The function $\theta_{\varepsilon} \in L^{\infty}\left(\Omega_{T}\right) \cap L^{2}\left(0, T ; H^{1}(\Omega)\right)$ is such that $0 \leq \theta_{\varepsilon}(x, t) \leq 1$ almost everywhere in $\Omega_{T}$.

We claim and prove in Section 3 that, as $\varepsilon$ tends to $0,\left(p_{\varepsilon}, \theta_{\varepsilon}\right)$ converges in some sense to a weak solution $(p, \theta)$ of the degenerate parabolic problem (1.1)-(1.2), (1.6)-(1.7).

REMARK 1.3. Let us give some physical motivations for such a problem with a vanishing mobility $\kappa=k / \mu$. On the one hand, the permeability may vanish in the domain. It models in particular percolation thresholds or Jacquin's validity limit of the Darcy law (see [5], Chapter 4). On the other hand, one cannot always ensure that the viscosity is bounded. We now describe the example of nuclear waste contamination. In this context, the fluid viscosity depends crucially on the temperature. The usual model used to include this dependence is (see [9])

$$
\mu(\theta)=\mu_{\text {ref }} \exp \left(\frac{1}{\theta-\theta_{\text {ref }}}\right),
$$

where $\mu_{\text {ref }}$ is some reference viscosity and $\theta_{\text {ref }}$ is some reference temperature. The viscosity is unbounded in the vicinity of $\theta_{\text {ref }}$. If we assume for the sake of simplicity that the viscosity vanishes only in an absolutely cold medium, that is, $\theta_{\text {ref }}=0$, note that the function

$$
\kappa(\theta)=\left\{\begin{array}{l}
\exp \left(-\frac{1}{\theta}\right) \text { if } 0<\theta \leq 1, \\
0 \text { if } \theta=0,
\end{array}\right.
$$

corresponds to the latter model of [9] and fulfills Assumption (1.4).

2. A priori estimates for the perturbated problem. We begin by deriving classical energy estimates on Problem (1.8)-(1.11).

Lemma 2.1. We claim that the following uniform estimates for the pressure hold true:

$$
\begin{aligned}
& \left\|p_{\varepsilon}\right\|_{L^{\infty}\left(0, T ; L^{2}(\Omega)\right)}+\left\|\kappa_{\varepsilon}\left(x, \theta_{\varepsilon}\right)^{1 / 2} \nabla p_{\varepsilon}\right\|_{\left(L^{2}\left(\Omega_{T}\right)\right)^{N}} \leq C, \\
& \left\|\kappa_{\varepsilon}\left(x, \theta_{\varepsilon}\right)^{-1 / 2} \underline{u}_{\varepsilon}\right\|_{\left(L^{2}\left(\Omega_{T}\right)\right)^{N}}+\left\|\underline{u}_{\varepsilon}\right\|_{\left(L^{2}\left(\Omega_{T}\right)\right)^{N}} \leq C .
\end{aligned}
$$

Furthermore, the function $\phi \partial_{t} p_{\varepsilon}$ is uniformly bounded in $L^{2}\left(0, T ;\left(H^{1}(\Omega)\right)^{\prime}\right)$. The sequence $\left(\theta_{\varepsilon}\right)$ is uniformly bounded in the space $L^{\infty}\left(\Omega_{T}\right) \cap L^{2}\left(0, T ; H^{1}(\Omega)\right)$. The dispersive 
term satisfies

$$
\left\|\left|\underline{u}_{\varepsilon}\right|^{1 / 2} \nabla \theta_{\varepsilon}\right\|_{\left(L^{2}\left(\Omega_{T}\right)\right)^{N}} \leq C .
$$

Proof. We multiply Eq. (1.8) by $p_{\varepsilon}$ and we integrate over $\Omega$. Integrating by parts, we obtain

$$
\frac{1}{2} \frac{d}{d t} \int_{\Omega} \phi\left|p_{\varepsilon}(\cdot, t)\right|^{2} d x+\int_{\Omega} \kappa_{\varepsilon} \nabla p_{\varepsilon} \cdot \nabla p_{\varepsilon} d x=\int_{\Omega}\left(q^{i}-q^{s}\right) p_{\varepsilon} d x
$$

We estimate the term on the right-hand side using the Cauchy-Schwarz and Young inequalities as follows:

$$
\left|\int_{\Omega}\left(q^{i}-q^{s}\right) p_{\varepsilon} d x\right| \leq C \int_{\Omega}\left|q^{i}-q^{s}\right|^{2} d x+C \int_{\Omega}\left|p_{\varepsilon}\right|^{2} d x \leq C+C \int_{\Omega}\left|p_{\varepsilon}\right|^{2} d x .
$$

Since $\phi(x) \geq \phi_{-}>0$ almost everywhere in $\Omega$, the latter relation and the Gronwall lemma give the first estimates of Lemma 2.1. The estimates on $\underline{u}_{\varepsilon}$ are a straightforward consequence since $\underline{u}_{\varepsilon}=-\kappa_{\varepsilon} \nabla p_{\varepsilon}$. Eq. (1.8) then directly implies that $\phi \partial_{t} p_{\varepsilon}$ is uniformly bounded in $L^{2}\left(0, T ;\left(H^{1}(\Omega)\right)^{\prime}\right)$.

We now consider Problem (1.9), (1.11). We recall that the temperature $\theta_{\varepsilon}$ satisfies $0 \leq \theta_{\varepsilon}(x, t) \leq 1$ almost everywhere in $\Omega_{T}$. The sequence $\left(\theta_{\varepsilon}\right)$ is then uniformly bounded in $L^{\infty}\left(\Omega_{T}\right)$. We now multiply Eq. (1.9) by $\theta_{\varepsilon}$ and we integrate over $\Omega$. We obtain

$$
\begin{aligned}
\frac{1}{2} \frac{d}{d t} \int_{\Omega} \phi\left|\theta_{\varepsilon}(\cdot, t)\right|^{2} d x & +\int_{\Omega} \mathcal{D}\left(\underline{u}_{\varepsilon}\right) \nabla \theta_{\varepsilon} \cdot \nabla \theta_{\varepsilon} d x \\
& +\int_{\Omega}\left(\underline{u}_{\varepsilon} \cdot \nabla \theta_{\varepsilon}\right) \theta_{\varepsilon} d x+\int_{\Omega} q^{i}\left|\theta_{\varepsilon}\right|^{2} d x=\int_{\Omega} q^{i} \theta_{\varepsilon} d x .
\end{aligned}
$$

Since $\theta_{\varepsilon}$ is uniformly bounded in $L^{\infty}\left(\Omega_{T}\right)$, we write, using the Cauchy-Schwarz and Young inequalities,

$$
\begin{aligned}
\left|\int_{\Omega}\left(\underline{u}_{\varepsilon} \cdot \nabla \theta_{\varepsilon}\right) \theta_{\varepsilon} d x\right| & \leq C \int_{\Omega}\left|\underline{u}_{\varepsilon}\right| d x+\frac{\phi_{-} \alpha_{t}}{2} \int_{\Omega}\left|\underline{u}_{\varepsilon}\right|\left|\nabla \theta_{\varepsilon}\right|^{2} d x \\
& \leq C(t)+\frac{\phi_{-} \alpha_{t}}{2} \int_{\Omega}\left|\underline{u}_{\varepsilon}\right|\left|\nabla \theta_{\varepsilon}\right|^{2} d x,
\end{aligned}
$$

where the quantity $C(t)$ is uniformly bounded in $L^{1}(0, T)$. The last term of the lefthand side of (2.1) is nonnegative. The right-hand side is bounded by a constant since $q^{i} \in L^{2}(\Omega)$ and $0 \leq \theta_{\varepsilon}(x, t) \leq 1$ a.e. in $\Omega_{T}$. Using Property (1.5), Relation (2.1) thus leads to

$$
\frac{1}{2} \frac{d}{d t} \int_{\Omega} \phi\left|\theta_{\varepsilon}(\cdot, t)\right|^{2} d x+\int_{\Omega} \phi_{-}\left(D_{m}+\frac{\alpha_{t}}{2}\left|\underline{u}_{\varepsilon}\right|\right)\left|\nabla \theta_{\varepsilon}\right|^{2} d x \leq C(t)+C .
$$

Bearing in mind that $\phi(x) \geq \phi_{-}>0$ a.e. in $\Omega$, we close this proof using the Gronwall lemma.

We now claim and prove the following compactness result.

LEMma 2.2. The sequence $\left(\theta_{\varepsilon}\right)$ is sequentially compact in $L^{2}\left(\Omega_{T}\right)$. 
Proof. We claim that the sequence $\partial_{t}\left(\phi \theta_{\varepsilon}\right)=\phi \partial_{t} \theta_{\varepsilon}$ is uniformly bounded in the space $L^{4 / 3}\left(0, T ;\left(W^{1,4}(\Omega)\right)^{\prime}\right)$. Indeed, multiplying Eq. (1.9) by a test function $\psi \in$ $L^{4}\left(0, T ; W^{1,4}(\Omega)\right)$ and integrating over $\Omega_{T}$, we obtain

$$
\begin{aligned}
\left\langle\phi \partial_{t} \theta_{\varepsilon}, \psi\right\rangle= & -\int_{\Omega_{T}}\left(\underline{u}_{\varepsilon} \cdot \nabla \theta_{\varepsilon}\right) \psi d x d t-\int_{\Omega_{T}} \mathcal{D}\left(\underline{u}_{\varepsilon}\right) \nabla \theta_{\varepsilon} \cdot \nabla \psi d x d t \\
& -\int_{\Omega_{T}} q^{i} \theta_{\varepsilon} \psi d x d t+\int_{\Omega_{T}} q^{i} \psi d x d t .
\end{aligned}
$$

The previous lemma ensures, in particular, that the sequence $\left|\underline{u}_{\varepsilon}\right| \nabla \theta_{\varepsilon}$ is uniformly bounded in $\left(L^{4 / 3}\left(\Omega_{T}\right)\right)^{N}$. Therefore,

$$
\begin{aligned}
\left|\left\langle\phi \partial_{t} \theta_{\varepsilon}, \psi\right\rangle\right| \leq \| & \left\|\underline{u}_{\varepsilon} \mid \nabla \theta_{\varepsilon}\right\|_{\left(L^{4 / 3}\left(\Omega_{T}\right)\right)^{N}}\|\psi\|_{L^{4}\left(\Omega_{T}\right)}+\phi_{+} D_{m}\left\|\nabla \theta_{\varepsilon}\right\|_{\left(L^{2}\left(\Omega_{T}\right)\right)^{N}}\|\nabla \psi\|_{\left(L^{2}\left(\Omega_{T}\right)\right)^{N}} \\
& +\phi_{+} \alpha_{l}\left\|\underline{u}_{\varepsilon} \mid \nabla \theta_{\varepsilon}\right\|_{\left(L^{4 / 3}\left(\Omega_{T}\right)\right)^{N}}\|\nabla \psi\|_{\left(L^{4}\left(\Omega_{T}\right)\right)^{N}} \\
& +\left\|q^{i}\right\|_{L^{2}(\Omega)}\left(1+\left\|\theta_{\varepsilon}\right\|_{L^{\infty}\left(\Omega_{T}\right)}\right)\|\psi\|_{L^{\infty}\left(0, T ; L^{2}(\Omega)\right)} \leq C\|\psi\|_{L^{4}\left(0, T ; W^{1,4}(\Omega)\right)} .
\end{aligned}
$$

Since $\phi \theta_{\varepsilon}$ is also bounded in $L^{2}\left(\Omega_{T}\right)$, the sequential compactness of $\left(\phi \theta_{\varepsilon}\right)$ in the space $L^{2}\left(0, T ;\left(H^{1}(\Omega)\right)^{\prime}\right)$ follows from a compactness argument of Aubin's type (see, for instance, [10]). Bearing in mind that $\theta_{\varepsilon}$ is uniformly bounded in $L^{2}\left(0, T ; H^{1}(\Omega)\right)$, we then can pass to the limit as $\varepsilon \rightarrow 0$ in the product $\left\langle\phi \theta_{\varepsilon}, \theta_{\varepsilon}\right\rangle$ of $L^{2}\left(0, T ;\left(H^{1}(\Omega)\right)^{\prime}\right) \times$ $L^{2}\left(0, T ; H^{1}(\Omega)\right)$. Since $\phi(x) \geq \phi_{-}>0$ a.e. in $\Omega$, the sequential compactness of $\theta_{\varepsilon}$ in $L^{2}\left(\Omega_{T}\right)$ follows.

At this step, we clearly do not have enough compactness results to pass to the limit in the fully nonlinear problem (1.8)-(1.11). One of the main difficulties is the lack of estimates on the pressure $p_{\varepsilon}$ due to the degeneracy of Eq. (1.8) as $\varepsilon$ tends to zero. We thus add the following result.

Lemma 2.3. For any $\eta>0$, the sequence $\phi\left(p_{\varepsilon} / \sqrt{p_{\varepsilon}^{2}+\eta}\right) \partial_{t} p_{\varepsilon}$ is uniformly bounded in $L^{1}\left(0, T ;\left(W^{1,4}(\Omega)\right)^{\prime}\right)$.

Proof. Let $\psi$ be a given function of $L^{\infty}\left(0, T ; W^{1,4}(\Omega)\right)$. We write the variational formulation of Eq. (1.8) for the test function $p_{\varepsilon} \psi\left(p_{\varepsilon}^{2}+\eta\right)^{-1 / 2}$ and we get

$$
\begin{aligned}
& \left\langle\phi p_{\varepsilon}\left(p_{\varepsilon}^{2}+\eta\right)^{-1 / 2} \partial_{t} p_{\varepsilon}, \psi\right\rangle=\int_{\Omega_{T}}\left(\underline{u}_{\varepsilon} \cdot \nabla \psi\right) p_{\varepsilon}\left(p_{\varepsilon}^{2}+\eta\right)^{1 / 2} d x d t \\
& \quad+\int_{\Omega_{T}}\left(\underline{u}_{\varepsilon} \cdot \nabla p_{\varepsilon}\right) \eta\left(p_{\varepsilon}^{2}+\eta\right)^{-3 / 2} \psi d x d t+\int_{\Omega_{T}}\left(q^{i}-q^{s}\right) p_{\varepsilon}\left(p_{\varepsilon}^{2}+\eta\right)^{-1 / 2} \psi d x d t .
\end{aligned}
$$

We note that the functions $p_{\varepsilon}\left(p_{\varepsilon}^{2}+\eta\right)^{-1 / 2}$ and $\eta\left(p_{\varepsilon}^{2}+\eta\right)^{-3 / 2}$ are uniformly bounded in $L^{\infty}\left(\Omega_{T}\right)$. With Lemma 2.1 , we can assert that $\underline{u}_{\varepsilon} \cdot \nabla p_{\varepsilon}$ is uniformly bounded in $L^{1}\left(\Omega_{T}\right)$. Using, in particular, the embedding $W^{1,4}(\Omega) \subset L^{\infty}(\Omega)$, we write the uniform estimate $\left|\left\langle\left(\phi p_{\varepsilon}\left(p_{\varepsilon}^{2}+\eta\right)^{-1 / 2}\right) \partial_{t} p_{\varepsilon}, \psi\right\rangle\right| \leq C\|\psi\|_{L^{\infty}\left(0, T ; W^{1,4}(\Omega)\right)}$. Lemma 2.3 is proved.

3. Convergence results as $\varepsilon \rightarrow 0$ and proof of Theorem 1.1. We now study the asymptotic behavior of Problem (1.8)-(1.11) as $\varepsilon$ tends to zero. The previous results let 
us assert the existence of functions $p \in L^{\infty}\left(0, T ; L^{2}(\Omega)\right), \underline{u} \in\left(L^{2}\left(\Omega_{T}\right)\right)^{N}, \theta \in L^{\infty}\left(\Omega_{T}\right) \cap$ $L^{2}\left(0, T ; H^{1}(\Omega)\right)$ such that, for extracted subsequences not relabeled for convenience,

$$
\begin{aligned}
& p_{\varepsilon} \rightarrow p \text { weakly in } L^{2}\left(\Omega_{T}\right), \quad \underline{u}_{\varepsilon} \rightarrow \underline{u} \text { weakly in }\left(L^{2}\left(\Omega_{T}\right)\right)^{N}, \\
& \theta_{\varepsilon} \rightarrow \theta \text { weakly* in } L^{\infty}\left(\Omega_{T}\right), \text { weakly in } L^{2}\left(0, T ; H^{1}(\Omega)\right), \text { and a.e. in } \Omega_{T} .
\end{aligned}
$$

These functions satisfy the following limit equations in $\Omega_{T}$ :

$$
\begin{aligned}
& \phi \partial_{t} p+\operatorname{div}(\underline{u})=q^{i}-q^{s}, \quad \underline{u}=-\overline{\kappa_{\varepsilon}\left(x, \theta_{\varepsilon}\right) \nabla p_{\varepsilon}}, \\
& \phi \partial_{t} \theta+\underline{u_{\varepsilon} \cdot \nabla \theta_{\varepsilon}}-\operatorname{div}\left(\phi\left(D_{m} \nabla \theta+\overline{\left.D_{p}\left(\underline{u}_{\varepsilon}\right) \nabla \theta_{\varepsilon}\right)}\right)+q^{i} \theta=q^{i},\right.
\end{aligned}
$$

where $\overline{f_{\varepsilon}}$ denotes the weak ad hoc limit of a sequence $\left(f_{\varepsilon}\right)$.

We have to exposit the previously unknown limits. To this aim, we recall the following compensated compactness result.

Lemma 3.1. Let $V, W$ and $V_{1}$ be Banach spaces such that $D(\Omega) \subset V \subset W \subset D^{\prime}(\Omega)$ and $V^{\prime} \subset V_{1}^{\prime} \subset D^{\prime}(\Omega)$, with continuous embedding, the embedding $V \subset W$ being compact. Let $\left(\alpha_{\varepsilon}\right)$ and $\left(\beta_{\varepsilon}\right)$ be sequences such that

$\left(\alpha_{\varepsilon}\right)$ is bounded in $L^{p}(0, T ; V), \alpha_{\varepsilon} \rightarrow \alpha$ weakly in $L^{p}(0, T ; W)$, for some $1<p<\infty$,

$\left(\beta_{\varepsilon}\right)$ is bounded in $L^{q}\left(0, T ; W^{\prime}\right), \beta_{\varepsilon} \rightarrow \beta$ weakly in $L^{q}\left(0, T ; W^{\prime}\right)$, for $q \geq p /(p-1)$,

$\left(\partial_{t} \beta_{\varepsilon}\right)$ is bounded in $L^{p_{1}}\left(0, T ; V_{1}^{\prime}\right)$, for some $p_{1} \geq 1$.

Then, up to extracted subsequences, the convergence $\alpha_{\varepsilon} \beta_{\varepsilon} \rightarrow \alpha \beta$ holds true in $D^{\prime}\left(\Omega_{T}\right)$.

This version of the div-curl lemma of Murat and Tartar (see [8, 11]) is given by A.V. Kazhikhov in [7, Lemma 6]). Amirat and Ziani already use it in [2] for the asymptotic study of a displacement model in porous media. Actually they study the asymptotic behavior as $D_{m} \rightarrow 0$ of a problem similar to (1.1)-(1.2) in the incompressible case $(\operatorname{div} \underline{u}=0)$ when the mobility is bounded and bounded below. They use Lemma 3.1 to pass to the limit in a term of the form $\theta_{\varepsilon} \nabla p_{\varepsilon}$ where $\theta_{\varepsilon}$ and $\nabla p_{\varepsilon}$ are both bounded sequences in $L^{2}$. We now apply this result in a different context. Indeed we aim to pass to the limit in the Darcy law $\underline{u}_{\varepsilon}=-\kappa_{\varepsilon}\left(x, \theta_{\varepsilon}\right) \nabla p_{\varepsilon}$ where we have an estimate for $\kappa_{\varepsilon}\left(x, \theta_{\varepsilon}\right)^{1 / 2} \nabla p_{\varepsilon}$ in $\left(L^{2}\right)^{N}$ but nothing for $\nabla p_{\varepsilon}$ alone. To overcome this difficulty, we re-apply the compactness argument of Lemma 3.1 several times to prove the following $\varepsilon$-limit result.

Lemma 3.2. The limit velocity is defined by the Darcy law $\underline{u}=-\kappa(x, c) \nabla p$.

Proof. Let us give the outline of the proof. The difficulty is the lack of estimates for $\nabla p_{\varepsilon}$. We thus write $\underline{u}_{\varepsilon}=-\kappa_{\varepsilon}\left(x, \theta_{\varepsilon}\right) \nabla p_{\varepsilon}=-\nabla\left(\kappa_{\varepsilon}\left(x, \theta_{\varepsilon}\right) p_{\varepsilon}\right)+p_{\varepsilon} \nabla \kappa_{\varepsilon}\left(x, \theta_{\varepsilon}\right)$. Getting a compactness result for $p_{\varepsilon}$ seems hopeless. But applying Lemma 3.1 several times, we prove the compactness of $\kappa_{\varepsilon}\left(x, \theta_{\varepsilon}\right)^{1 / 2} p_{\varepsilon}$. We then state that it is sufficient to pass to the limit in the latter relation.

Let $\eta>0$ be a fixed real. We set $P_{\varepsilon}=\sqrt{p_{\varepsilon}^{2}+\eta}$ and $\beta_{\varepsilon}=\phi P_{\varepsilon}$. By Lemma 2.1, the sequence $\left(\beta_{\varepsilon}\right)$ is uniformly bounded in $L^{\infty}\left(0, T ; L^{2}(\Omega)\right)$. Let us denote $\phi P$ as its weak limit:

$$
\beta_{\varepsilon} \rightarrow \phi P \text { weakly* in } L^{\infty}(0, T ; W), \quad W=L^{2}(\Omega)=W^{\prime}
$$


By Lemma $2.3,\left(\partial_{t} \beta_{\varepsilon}\right)$ is bounded in $L^{1}\left(0, T ; V_{1}^{\prime}\right), V_{1}=W^{1,4}(\Omega)$. We note that $P_{\varepsilon}(x, t) \geq$ $\eta^{1 / 2}>0$ a.e. in $\Omega_{T}$.

On the one hand, we set $\alpha_{\varepsilon}^{1}=\kappa_{\varepsilon}\left(x, \theta_{\varepsilon}\right) P_{\varepsilon}\left(P_{\varepsilon}+1\right)^{-1}$. This quantity is clearly bounded in $L^{\infty}\left(\Omega_{T}\right)$. With Lemma 2.1 , we check that $\nabla \alpha_{\varepsilon}^{1}$ is uniformly bounded in $\left(L^{2}\left(\Omega_{T}\right)\right)^{N}$. We thus assert that $\alpha_{\varepsilon}^{1}$ is bounded in $L^{2}(0, T ; V)$ with $V=H^{1}(\Omega)$ compactly embedded in $W$. We apply Lemma 3.1 and get $\alpha_{\varepsilon}^{1} \beta_{\varepsilon} \rightarrow \alpha^{1} \beta$ in $D^{\prime}\left(\Omega_{T}\right)$. Since $\theta_{\varepsilon} \rightarrow \theta$ almost everywhere in $\Omega_{T}$, this reads

$$
\phi \kappa_{\varepsilon}\left(x, \theta_{\varepsilon}\right) P_{\varepsilon}\left(P_{\varepsilon}+1\right)^{-1} P_{\varepsilon} \rightarrow \phi \kappa(x, \theta) \overline{P_{\varepsilon}\left(P_{\varepsilon}+1\right)^{-1}} P .
$$

The same idea works with $\phi\left(P_{\varepsilon}+1\right)$ instead of $\beta_{\varepsilon}$ and gives

$$
\phi \kappa_{\varepsilon}\left(x, \theta_{\varepsilon}\right) P_{\varepsilon}=\phi \kappa_{\varepsilon}\left(x, \theta_{\varepsilon}\right) P_{\varepsilon}\left(P_{\varepsilon}+1\right)^{-1}\left(P_{\varepsilon}+1\right) \rightarrow \phi \kappa(x, \theta) \overline{P_{\varepsilon}\left(P_{\varepsilon}+1\right)^{-1}}(P+1) .
$$

Since the a.e. convergence of $\theta_{\varepsilon}$ to $\theta$ gives $\phi \kappa_{\varepsilon}\left(x, \theta_{\varepsilon}\right) P_{\varepsilon} \rightarrow \phi \kappa(x, \theta) P$ weakly* in the space $L^{\infty}\left(0, T ; L^{2}(\Omega)\right)$, we conclude that $\kappa(x, \theta) \frac{\frac{P_{\varepsilon}}{P_{\varepsilon}+1}}{P_{\varepsilon}}=\kappa(x, \theta) \frac{P}{P+1}$. Bearing in mind that $\phi(x) \geq \phi_{-}>0$ a.e. in $\Omega$, Relation (3.1) is

$$
\kappa_{\varepsilon}\left(x, \theta_{\varepsilon}\right) P_{\varepsilon}^{2}\left(P_{\varepsilon}+1\right)^{-1} \rightarrow \kappa(x, \theta) P^{2}(P+1)^{-1} .
$$

On the other hand, we set $\alpha_{\varepsilon}^{2}=\kappa_{\varepsilon}\left(x, \theta_{\varepsilon}\right)^{1 / 2}\left(P_{\varepsilon}+1\right)^{-1 / 2}$. Because of Assumption (1.4), the sequence $\alpha_{\varepsilon}^{2}$ satisfies the same properties as $\alpha_{\varepsilon}^{1}$. We thus assert with Lemma 3.1 that $\alpha_{\varepsilon}^{2} \beta_{\varepsilon} \rightarrow \alpha^{2} \beta$ in $D^{\prime}\left(\Omega_{T}\right)$; that is,

$$
\phi \kappa_{\varepsilon}\left(x, \theta_{\varepsilon}\right)^{1 / 2}\left(P_{\varepsilon}+1\right)^{-1 / 2} P_{\varepsilon} \rightarrow \phi \kappa(x, \theta)^{1 / 2} \overline{\left(P_{\varepsilon}+1\right)^{-1 / 2}} P .
$$

We recall that $P(x, t) \geq \eta^{1 / 2}>0$ almost everywhere in $\Omega_{T}$. Using the convexity of the function $x \mapsto(x+1)^{-1 / 2}$, we claim following [11] that

$$
\overline{\left(P_{\varepsilon}+1\right)^{-1 / 2}} \geq(P+1)^{-1 / 2} \quad \text { and } \quad \kappa(x, \theta)^{1 / 2} \overline{\left(P_{\varepsilon}+1\right)^{-1 / 2}} P \geq \kappa(x, \theta)^{1 / 2} P(P+1)^{-1 / 2} \text {. }
$$

Studying the concavity of $x \mapsto x / \sqrt{x+1}$ in $\mathbb{R}_{+}$, we conclude that $\overline{P_{\varepsilon}\left(P_{\varepsilon}+1\right)^{-1 / 2}} \leq$ $P(P+1)^{-1 / 2}$ and

$$
\overline{\kappa_{\varepsilon}\left(x, \theta_{\varepsilon}\right)^{1 / 2} P_{\varepsilon}\left(P_{\varepsilon}+1\right)^{-1 / 2}}=\kappa(x, \theta)^{1 / 2} \overline{P_{\varepsilon}\left(P_{\varepsilon}+1\right)^{-1 / 2}} \leq \kappa(x, \theta)^{1 / 2} P(P+1)^{-1 / 2} .
$$

Combining (3.3)-(3.5) with $\phi(x) \geq \phi_{-}>0$ a.e. in $\Omega$, we get

$$
\kappa_{\varepsilon}\left(x, \theta_{\varepsilon}\right)^{1 / 2} P_{\varepsilon}\left(P_{\varepsilon}+1\right)^{-1 / 2} \rightarrow \kappa(x, \theta)^{1 / 2} P(P+1)^{-1 / 2} .
$$

Now we exploit Relations (3.2) and (3.6). A consequence is the a.e. convergence of $\kappa_{\varepsilon}\left(x, \theta_{\varepsilon}\right) P_{\varepsilon}^{2}\left(P_{\varepsilon}+1\right)^{-1}$ to $\kappa(x, \theta) P^{2}(P+1)^{-1}$. Since $P_{\varepsilon}+1 \rightarrow P+1$, we conclude that

$$
\kappa_{\varepsilon}\left(x, \theta_{\varepsilon}\right) P_{\varepsilon}^{2}=\kappa_{\varepsilon}\left(x, \theta_{\varepsilon}\right) P_{\varepsilon}^{2}\left(P_{\varepsilon}+1\right)^{-1}\left(P_{\varepsilon}+1\right) \rightarrow \kappa(x, \theta) P^{2} .
$$

Since we already know that $\kappa_{\varepsilon}\left(x, \theta_{\varepsilon}\right)^{1 / 2} P_{\varepsilon} \rightarrow \kappa(x, \theta)^{1 / 2} P$ in $L^{2}\left(\Omega_{T}\right)$, the latter relation proves that $\kappa_{\varepsilon}\left(x, \theta_{\varepsilon}\right)^{1 / 2} P_{\varepsilon} \rightarrow \kappa(x, \theta)^{1 / 2} P$ almost everywhere in $\Omega_{T}$. It follows that

$$
\kappa_{\varepsilon}\left(x, \theta_{\varepsilon}\right) P_{\varepsilon}^{2}=\kappa_{\varepsilon}\left(x, \theta_{\varepsilon}\right)\left(p_{\varepsilon}^{2}+\eta\right) \rightarrow \kappa(x, \theta)\left(\overline{p_{\varepsilon}^{2}}+\eta\right) \text { a.e. in } \Omega_{T}
$$

and, taking the square root of the latter relation,

$$
\kappa_{\varepsilon}\left(x, \theta_{\varepsilon}\right)^{1 / 2} p_{\varepsilon} \rightarrow \kappa(x, \theta)^{1 / 2} p \text { a.e. in } \Omega_{T} .
$$


We now turn back to the Darcy law. We write

$$
\begin{aligned}
& p_{\varepsilon} \nabla \kappa_{\varepsilon}\left(x, \theta_{\varepsilon}\right)=p_{\varepsilon} \nabla_{x} \kappa_{\varepsilon}\left(x, \theta_{\varepsilon}\right)+D_{\theta} \kappa_{\varepsilon}\left(x, \theta_{\varepsilon}\right) p_{\varepsilon} \nabla \theta_{\varepsilon} \\
& \quad=\kappa_{\varepsilon}\left(x, \theta_{\varepsilon}\right)^{1 / 2} p_{\varepsilon} \frac{1}{\kappa_{\varepsilon}\left(x, \theta_{\varepsilon}\right)^{1 / 2}} \nabla_{x} \kappa_{\varepsilon}\left(x, \theta_{\varepsilon}\right)+\kappa_{\varepsilon}\left(x, \theta_{\varepsilon}\right)^{1 / 2} p_{\varepsilon} \frac{D_{\theta} \kappa_{\varepsilon}\left(x, \theta_{\varepsilon}\right)}{\kappa_{\varepsilon}\left(x, \theta_{\varepsilon}\right)^{1 / 2}} \nabla \theta_{\varepsilon} .
\end{aligned}
$$

Using (1.4) and the a.e. convergence of $\theta_{\varepsilon}$ to $\theta$, we assert that $\kappa_{\varepsilon}\left(x, \theta_{\varepsilon}\right)^{-1 / 2} \nabla_{x} \kappa_{\varepsilon}\left(x, \theta_{\varepsilon}\right) \rightarrow$ $2 \nabla_{x} \kappa(x, \theta)^{1 / 2}$ and $\kappa_{\varepsilon}\left(x, \theta_{\varepsilon}\right)^{-1 / 2} D_{\theta} \kappa_{\varepsilon}\left(x, \theta_{\varepsilon}\right) \rightarrow 2 D_{\theta} \kappa(x, \theta)^{1 / 2}$ a.e. in $\Omega_{T}$. Moreover, using (3.7), we conclude that

$$
p_{\varepsilon} \nabla \kappa_{\varepsilon}\left(x, \theta_{\varepsilon}\right) \rightarrow 2 \kappa(x, \theta)^{1 / 2} p \nabla \kappa(x, \theta)^{1 / 2}=p \nabla \kappa(x, \theta)
$$

weakly in $\left(L^{1}\left(\Omega_{T}\right)\right)^{N}$. Finally, writing $\kappa_{\varepsilon}\left(x, \theta_{\varepsilon}\right) \nabla p_{\varepsilon}=-p_{\varepsilon} \nabla \kappa_{\varepsilon}\left(x, \theta_{\varepsilon}\right)+\nabla\left(\kappa_{\varepsilon}\left(x, \theta_{\varepsilon}\right) p_{\varepsilon}\right)$, we obtain

$$
\kappa_{\varepsilon}\left(x, \theta_{\varepsilon}\right) \nabla p_{\varepsilon} \rightarrow \kappa(x, \theta) \nabla p \quad \text { weakly in }\left(L^{2}\left(\Omega_{T}\right)\right)^{N} .
$$

This ends the proof of Lemma 3.2.

It remains to pass to the limit in the nonlinearities of the temperature equation (1.9). We first prove the following compactness result.

Lemma 3.3. We claim that, up to extracted subsequences, $p_{\varepsilon} \rightarrow p$ a.e. in $\Omega_{T}$ and

$$
\kappa_{\varepsilon}\left(x, \theta_{\varepsilon}\right)^{1 / 2} \nabla p_{\varepsilon} \rightarrow \kappa(x, \theta)^{1 / 2} \nabla p \quad \text { strongly in }\left(L^{2}\left(\Omega_{T}\right)\right)^{N} \text { and a.e. in } \Omega_{T} .
$$

Proof. We multiply Eq. (1.8) by $p_{\varepsilon}$ and its limit equation by $p$. Integrating by parts over $\Omega_{t}=\Omega \times(0, t)$ for any $t \in(0, T)$, we obtain

$$
\begin{aligned}
& \frac{1}{2} \int_{\Omega} \phi\left|p_{\varepsilon}(\cdot, t)\right|^{2} d x-\frac{1}{2} \int_{\Omega} \phi\left|p_{o}(\cdot)\right|^{2} d x \\
&+\int_{\Omega_{t}} \kappa_{\varepsilon}\left(x, \theta_{\varepsilon}\right) \nabla p_{\varepsilon} \cdot \nabla p_{\varepsilon} d x d s=\int_{\Omega_{t}}\left(q^{i}-q^{s}\right) p_{\varepsilon} d x d s, \\
& \frac{1}{2} \int_{\Omega} \phi|p(\cdot, t)|^{2} d x-\frac{1}{2} \int_{\Omega} \phi\left|p_{o}(\cdot)\right|^{2} d x \\
&+\int_{\Omega_{t}} \kappa(x, \theta) \nabla p \cdot \nabla p d x d s=\int_{\Omega_{t}}\left(q^{i}-q^{s}\right) p d x d s .
\end{aligned}
$$

Thus, using the weak $L^{2}$-convergence of $p_{\varepsilon}$ to $p$, we claim that for any $t \in(0, T)$,

$$
\begin{aligned}
& \lim _{\varepsilon \rightarrow 0} \int_{\Omega} \phi\left|p_{\varepsilon}(\cdot, t)\right|^{2} d x=\int_{\Omega} \phi|p(\cdot, t)|^{2} d x, \\
& \lim _{\varepsilon \rightarrow 0} \int_{\Omega_{t}} \kappa_{\varepsilon}\left(x, \theta_{\varepsilon}\right) \nabla p_{\varepsilon} \cdot \nabla p_{\varepsilon} d x d s=\int_{\Omega_{t}} \kappa(x, \theta) \nabla p \cdot \nabla p d x d s .
\end{aligned}
$$

We begin by exploiting the first relation of (3.8). It allows us to compute

$$
\lim _{\varepsilon \rightarrow 0} \int_{\Omega} \phi\left(p_{\varepsilon}-p\right)^{2} d x=0 .
$$

Since $\phi(x) \geq \phi_{-}>0$ almost everywhere in $\Omega$, it proves that $p_{\varepsilon} \rightarrow p$ strongly in $L^{2}\left(\Omega_{T}\right)$ and thus almost everywhere in $\Omega_{T}$. We similarly exploit the second part of (3.8). It follows that

$$
\lim _{\varepsilon \rightarrow 0} \int_{\Omega_{t}}\left(\kappa_{\varepsilon}\left(x, \theta_{\varepsilon}\right)^{1 / 2} \nabla p_{\varepsilon}-\kappa(x, \theta)^{1 / 2} \nabla p\right) \cdot\left(\kappa_{\varepsilon}\left(x, \theta_{\varepsilon}\right)^{1 / 2} \nabla p_{\varepsilon}-\kappa(x, \theta)^{1 / 2} \nabla p\right) d x d s=0 .
$$


This ends the proof of Lemma 3.3.

We now have the tools to pass to the limit $\varepsilon \rightarrow 0$ in the temperature equation (1.9). We write the nonlinearities containing the Darcy velocity $\underline{u}_{\varepsilon}$ under the following forms:

$$
\begin{aligned}
& \underline{u}_{\varepsilon} \cdot \nabla \theta_{\varepsilon}=\operatorname{div}\left(\underline{u}_{\varepsilon} \theta_{\varepsilon}\right)-\theta_{\varepsilon} \operatorname{div}\left(\underline{u}_{\varepsilon}\right), \\
& \left|\underline{u}_{\varepsilon}\right| \mathcal{E}\left(\underline{u}_{\varepsilon}\right)_{i j}=\kappa_{\varepsilon}\left(x, \theta_{\varepsilon}\right)^{1 / 2} \frac{\left(\kappa_{\varepsilon}\left(x, \theta_{\varepsilon}\right)^{1 / 2} \nabla p_{\varepsilon}\right)_{i}\left(\kappa_{\varepsilon}\left(x, \theta_{\varepsilon}\right)^{1 / 2} \nabla p_{\varepsilon}\right)_{j}}{\left|\kappa_{\varepsilon}\left(x, \theta_{\varepsilon}\right)^{1 / 2} \nabla p_{\varepsilon}\right|} .
\end{aligned}
$$

The previous compactness results are sufficient to pass to the limit in these expressions.

Theorem 1.1 is proved.

\section{REFERENCES}

[1] Y. Amirat, K. Hamdache, and A. Ziani. Mathematical analysis for compressible miscible displacement models in porous media. Math. Models Methods Appl. Sci., 6(6):729-747, 1996. MR1404826 (97g:76086)

[2] Y. Amirat and A. Ziani. Asymptotic behavior of the solutions of an elliptic-parabolic system arising in flow in porous media. Z. Anal. Andwendungen, 23(2):335-351, 2004. MR2085294 (2007d:35215)

[3] J. Bear. Dynamics of Fluids in Porous Media. American Elsevier, 1972.

[4] C. Choquet. Existence result for a radionuclide transport model with an unbounded viscosity. $J$. Math. Fluid Mech., 6(4):365-388, 2004. MR2101887 (2005m:35142)

[5] G. de Marsily. Hydrogéologie quantitative. Masson, 1981.

[6] M. Kaviany. Principles of heat transfer in porous media. Springer, 1999.

[7] A.V. Kazhikhov. Recent developments in the global theory of two-dimensional compressible NavierStokes equations. Seminar on Mathematical Sciences, Keio University, 25, 1998. MR1600212 (98k:35150)

[8] F. Murat. Compacité par compensation. Ann. Scuola Norm. Sup. Pisa, 5:489-507, 1978. MR.506997 (80h:46043a)

[9] M. Reeves and R.M. Cranwell. User's manual for the Sandia Waste-Isolation Flow and Transport model (SWIFT). Release 4.81. Sandia Report Nureg/Cr-2324, SAND81-2516, GF, Sandia National Laboratories, Albuquerque, 1981.

[10] J. Simon. Compact sets in the space $L^{p}(0, T ; B)$. Ann. Math. Pura Appl., IV:65-96, 1987. MR916688 (89c:46055)

[11] L. Tartar. Compensated compactness and applications to P.D.E. In R.J. Knops, editor, Non Linear Analysis and Mechanics, Heriot-Watt Symposium, Research Notes in Math., volume 4 (39), pages 136-212. Pitman Press, 1979. MR584398 (81m:35014) 